\title{
Altered activation and connectivity of the supplementary motor cortex at motor initiation in Parkinson's disease patients with freezing
}

\author{
Brugger, Florian ; Wegener, Regina ; Walch, Julia ; Galovic, Marian ; Hägele-Link, Stefan ; Bohlhalter,
} Stephan ; Kägi, Georg

\begin{abstract}
OBJECTIVE Motor initiation failure is a key feature of freezing of gait (FOG) due to Parkinson's disease (PD). The supplementary motor cortex (SMC) plays a central role in its pathophysiology. We aimed at investigating SMC activation, connectivity and plasticity with regard to motor initiation in FOG. METHODS Twelve patients with FOG and eleven without FOG underwent a multimodal electrophysiological evaluation of SMC functioning including the Bereitschaftspotential and movement-related desynchronisation of cortical beta oscillations. SMC plasticity was modulated by intermittent theta burst stimulation (iTBS) and its impact on gait initiation was assessed by a three-dimensional gait analysis. RESULTS Prior to volitional movements the Bereitschaftspotential was smaller and beta power was less strongly attenuated over the SMC in patients with FOG compared to those without. Pre-motor coherence between the SMC and the primary motor cortex in the beta frequency range was also stronger in patients with FOG. iTBS resulted in a relative deterioration of gait initiation. CONCLUSIONS Reduced activation of the SMC along with increased SMC connectivity in the beta frequency range hinder a flexible shift of the motor set as it is required for gait initiation. SIGNIFICANCE Altered SMC functioning plays an important role for motor initiation failure in PD-related FOG.
\end{abstract}

DOI: https://doi.org/10.1016/j.clinph.2020.05.023

Posted at the Zurich Open Repository and Archive, University of Zurich

ZORA URL: https://doi.org/10.5167/uzh-192439

Journal Article

Accepted Version

Originally published at:

Brugger, Florian; Wegener, Regina; Walch, Julia; Galovic, Marian; Hägele-Link, Stefan; Bohlhalter, Stephan; Kägi, Georg (2020). Altered activation and connectivity of the supplementary motor cortex at motor initiation in Parkinson's disease patients with freezing. Clinical Neurophysiology, 131(9):2171-2180. DOI: https://doi.org/10.1016/j.clinph.2020.05.023 


\title{
Altered supplementary motor cortex functioning in patients with freezing of gait due to Parkinson's disease
}

Florian Brugger ${ }^{1,4}$, Regina Wegener ${ }^{2,3}$, Julia Walch ${ }^{1}$, Marian Galovic ${ }^{1,4,5}$, Stefan HägeleLink $^{1}$, Stephan Bohlhalter ${ }^{4}$, Georg Kägi ${ }^{1}$

\author{
${ }^{1}$ Department of Neurology, Kantonsspital St. Gallen, Switzerland \\ ${ }^{2}$ Laboratory for Motion Analysis, Department of Paediatric Orthopaedics, Children's \\ Hospital of Eastern Switzerland, St. Gallen, Switzerland \\ ${ }^{3}$ Department of Orthopaedics and Traumatology, Kantonsspital St. Gallen, \\ Switzerland \\ ${ }^{4}$ UCL Institute of Neurology, London, United Kingdom \\ ${ }^{5}$ Department of Neurology, University Hospital Zurich, Switzerland \\ ${ }^{6}$ Neurocenter, Luzerner Kantonsspital, Luzern, Switzerland
}

Correspondence to:

Dr. Florian Brugger

Department of Neurology

Kantonsspital St. Gallen

Rorschacherstrasse 95

9007 St. Gallen

+41714941657

florian.brugger@kssg.ch 
Word count: 4726 words (incl. references)

Number of tables: 2

Number of figures: 3

References: 63

Supplementary material: 1

Keywords: Parkinson's disease, freezing of gait, beta oscillations, Bereitschaftspotential, TMS

Financial disclosures: This study has been supported by a grant from the Swiss Parkinson Association (Parkinson Schweiz). 


\section{Financial disclosures:}

Florian Brugger: Employment xx; consultancies xx; stock ownership xx; honoraria xx; paid expert testimony $\mathrm{xx}$; patent registrations $\mathrm{xx}$; grants and other funding $\mathrm{xx}$;

Regina Wegener: Employment xx; consultancies xx; stock ownership xx; honoraria xx; paid expert testimony $\mathrm{xx}$; patent registrations $\mathrm{xx}$; grants and other funding $\mathrm{xx}$;

Julia Walch: Employment xx; consultancies xx; stock ownership xx; honoraria xx; paid expert testimony $\mathrm{xx}$; patent registrations $\mathrm{xx}$; grants and other funding $\mathrm{xx}$;

Marian Galovic: Employment University Hospital Zurich, Switzerland; consultancies None; stock ownership None; honoraria None; paid expert testimony None; patent registrations None; grants and other funding Epilepsy Research UK;

Stefan Hägele-Link: Employment xx; consultancies xx; stock ownership xx; honoraria xx; paid expert testimony $\mathrm{xx}$; patent registrations $\mathrm{xx}$; grants and other funding $\mathrm{xx}$;

Stephan Bohlhalter: Employment xx; consultancies xx; stock ownership xx; honoraria xx; paid expert testimony $\mathrm{xx}$; patent registrations $\mathrm{xx}$; grants and other funding $\mathrm{xx}$;

Georg Kägi: Employment xx; consultancies xx; stock ownership xx; honoraria xx; paid expert testimony $\mathrm{xx}$; patent registrations $\mathrm{xx}$; grants and other funding $\mathrm{xx}$; 


\section{Abstract (200 words)}

Objective: Gait ignition failure is a key feature of freezing of gait (FOG) due to Parkinson's disease (PD). A central role of altered supplementary motor cortex (SMC) functioning has been discussed previously. We aimed at investigating SMC functioning at gait initiation in PD-related FOG.

Method: Twelve patients with FOG and eleven without FOG underwent a multimodal electrophysiological evaluation of SMC functioning including the Bereitschaftspotential and movement-related desynchronisation of cortical beta oscillations. SMC plasticity was modulated by repeated transcranial magnetic stimulation (rTMS) and its impact on gait initiation was assessed by a three-dimensional gait analysis.

Results: Prior to volitional movements the Bereitschaftspotential was smaller and beta power was less strongly attenuated over the SMC in patients with FOG compared to those without. Pre-motor coherence between the SMC and the primary motor cortex in the beta frequency range was also stronger in patients with FOG. rTMS resulted in a relative deterioration of gait initiation.

Conclusion: There are profound quantitative and qualitative alterations of SMC functioning in PD patients with FOG. Our results suggest a malfunctioning of the motor network involved in uncued movements in PD-related FOG.

Significance: Altered SMC functioning plays an important role for gait ignition failure in PDrelated FOG. 


\section{Highlights ( $<85$ characters incl. spaces):}

The Bereitschaftspotential is smaller in patients with freezing of gait

Beta oscillations are increased prior to volitional movements in freezers.

Repetitive transcranial magnetic stimulation leads to deteriorated gait initiation. 


\section{Introduction}

Freezing of gait (FOG) is defined as an episodic inability of forward progression of the feet despite the intention to walk along with the subjective feeling of being glued to the floor [1], mainly when initiating gait, passing narrow spaces or during turning for directional changes [2]. OFF-FOG and ON-FOG are distinct forms with probably different underlying pathophysiology [3]. FOG also features several interepisodic gait abnormalities including disturbed bilateral leg coordination with increased gait asymmetry, increased step time variability, higher cadence along with reduced step length and longer double limb support time as well as higher variability of the initial swing side at gait initiation $[1,4,5]$.

Freezing may not be confined to gait, but can also affect other motor programs such as finger movements or speech $[6,7]$. Freezing phenomena are thought to occur due to a transient breakdown of a hierarchically organized neuronal network consisting of various cortical and subcortical structures [8]. Among the cortical areas the supplementary motor cortex (SMC) is assumed to play a central role for motor control. It is part of a neuronal network engaged in conceptualizing complex, self-guided (uncued) movements and interlimb coordination [9]. The Bereitschaftspotential (BP), a negative cortical potential, arising within 1.0-1.5 sec before volitional movements over the SMC underpins its contribution to movement initiation [10]. The BP presumably reflects preparatory cognitive processes before motor onset [9-11]. SMC lesions lead to deficient motor initiation, particularly of self-guided movements, including gait abnormalities and are accompanied by a loss of the BP [9, 12-14].

A central role of the SMC in PD-related FOG is supported by fMRI studies which revealed reduced SMC activation during imaginary walking tasks or antiphasic movements of lower limbs $[15,16]$ as well as during motor blocks on a stepping task akin freezing [17-19]. FOG also correlates with white matter abnormalities including altered projections between the SMC and subcortical structures [7, 20, 21]. However, these techniques could not establish a causal relationship between FOG and SMC abnormalities and they suffered from low 
temporal resolution. In particular, it is not clear whether SMC underactivation has a detrimental effect on FOG or whether it is part of compensatory mechanisms. Here, we analyzed the contribution of the SMC to freezing of gait with a multimodal approach using EEG and repetitive transcranial magnetic stimulation (rTMS).

Firstly, we harnessed the high temporal resolution of EEG to assess the BP, beta oscillations, and cortico-cortical coherence (as a measure of network connectivity) in PD patients with versus those without FOG. Beta oscillations are regarded as a local idling rhythm that preserves the current motor state [22]. The attenuation of these rhythms physiologically precedes voluntary movements in healthy persons [23]. In PD several studies have shown disturbed beta desynchronisation of oscillations in the beta frequency range prior to volitional movements in the basal ganglia as well as over cortical areas including the SMC [24, 25], that is associated with motor features such as bradykinesia, rigidity and motor blocks of upper limb movements $[22,26]$. We expected a more profound alteration of the neuronal activity within the SMC in PD patients with FOG, as reflected by a decreased BP and a more severely disrupted beta desynchronisation before the initiation of voluntary movements. We also expected differences in cortico-cortical coherence between the SMC and other motor network hubs such as the primary motor cortex and the lateral premotor cortex.

Secondly, we used rTMS to non-invasively modulate the activation of SMC and measure its impact on gait parameters. We hypothesiszed that excitatory rTMS over the SMC would improve gait initiation in PD patients with FOG, thus suggesting a causal relationship between SMC activation and FOG.

\section{Methods}

\section{Participants}

12 PD patients with predominant OFF-FOG (PD+FOG) and 11 without FOG (PD-FOG) matched for age and sex were included (see table 1). Participants were able to complete 
walking trails of $10 \mathrm{~m}$ length without assistance (i.e. Hoehn and Yahr stadium <4). Patients were allocated to the PD+FOG group if they rated $\geq 1$ points at item 3 of the Freezing of Gait Questionnaire (FOG-Q), a reliable commonly used rating scale [27], and if an experienced rater (F.B.) classified the clinical history as conclusive. Exclusion criteria were atypical Parkinsonism, any contraindication for TMS, deep brain stimulation or dementia (Mini Mental Status Examination <24, Frontal Assessment Battery <9). Participants with neurological conditions other than PD (e.g. stroke) or orthopedic conditions that could have interfered with their ability to walk (e.g. joint replacements, leg difference $>2 \mathrm{~cm}$ ) were also excluded. All participants were naïve for rTMS. PD medication had to be kept unchanged for $>4$ weeks prior to inclusion and for the whole study period. The local ethic committee approved this study. All participants gave their written informed consent prior to study inclusion.

\section{Study design}

Part I) Subjects were scheduled for two recordings of the BP on the same day. The first recording was done in the $\mathrm{OFF}$, the second recording one hour after having taken their regular PD medication (i.e. in the ON). Part II) All participants were investigated by a threedimensional gait analysis (3DGA). PD-FOG were assessed in the OFF at one single visit, whilst PD+FOG had to come in for two visits in the OFF separated by 1-4 weeks. In PD+FOG the 3DGA was repeated twice at each of these visits, once before and once immediately after a single rTMS session. Real rTMS or sham stimulation were applied in a single-blind, randomized cross-over design (for further details see supplementary material).

\section{Clinical assessment}

At screening PD patients were assessed by the Unified Parkinson Disease Rating Scales I-III (UPDRS), Hoehn and Yahr stadium, FOG-Q, Frontal Assessment Battery (FAB) and Mini 
Mental Status Examination (MMSE). The UPDRS III scores were obtained in PD+FOG twice at each visit of part II, once before and once after the stimulation session, in PD-FOG just once before the 3DGA. Each examination was recorded by a video camera. A blinded rater (J.W.) later scored all UPDRS items apart from rigidity. The latter scores were obtained unblinded (F.B).

\section{Bereitschaftspotential and beta desynchronisation}

Participants were seated in a chair with surface EEG electrodes placed over the supplementary motor cortex $(\mathrm{Cz}, \mathrm{Fz})$, the primary motor cortex $(\mathrm{C} 3, \mathrm{C} 4)$ and the dorsolateral prefrontal cortex (F3, F4) according to the 10-20-EEG system with the ground electrode fixed at the ear lobe (impedance: $<5 \mathrm{k} \Omega$, sampling frequency: $500 \mathrm{~Hz}$ ) [25, 28, 29]. Participants performed repetitive middle finger extensions of $0.5-1 \mathrm{sec}$ duration in a self-paced manner with an intermovement interval of $\sim 6-7 \mathrm{sec}$. To ensure uncued, self-initiated movements, the participants were not allowed to use any rhythmical encoding (e.g. counting). Surface electrodes were placed over the finger extensors and muscle activity was recorded by the virtue of electromyography (EMG) to determine the onset of the finger movements. Onset of each movement was determined manually after inspection of the corresponding EEG segment for artefacts. The BP was calculated offline (MATLAB, MathWorks, Natick, United States) by averaging $\geq 40$ artefact-free EEG segments lasting from 2.5 seconds before to 0.75 second after motor onset (dual-pass bandpass filter: $0.15-15 \mathrm{~Hz}$, baseline corrected). Comparable levodopa equivalence doses (LED) were given in both patient groups to induce the ON (PDFOG: $482.7 \pm 242.7$ vs. PD+FOG 353.6 $\pm 160.8, \mathrm{p}=0.152)$.

For the calculation of event-related beta desynchronisation EEG segments before movement initiation and in the intermovement interval i.e. $>1 \mathrm{sec}$ after the preceding and $>2.5 \mathrm{sec}$ before the next finger movement, were selected. The EEG segments were band-pass filtered in the beta frequency range $(13-30 \mathrm{~Hz})$ and were analyzed by a discrete Fast Fourier transform 
(FFT). A cosine curve was superimposed on the EEG segments to avoid edge effects during FFT (i.e. hanning filter). Power of each beta range during the intermovement interval and immediately before voluntary finger movements was calculated as area under the curve (AUC). Beta desynchronisation was calculated as described elsewhere [24, 25]:

$\left(\mathrm{AUC}_{\text {rest }}-\mathrm{AUC}_{\text {premovement }}\right) / \mathrm{AUC}_{\text {rest }} * 100$. Coherence between the different electrodes over the hemisphere contralateral to the moved limb was calculated by using Neurospec20, a freely available MATLAB routine [30]. Changes in coherence between the motor cortex (C3/4) and premotor cortices were calculated in analogy to beta desynchronisation as defined above. For data analysis $\mathrm{C} 3$ and $\mathrm{C} 4$ were renamed in relation to the side of the moved finger. Thus the terms ' $\mathrm{Cc}$ ' and ' $\mathrm{Fc}$ ' corresponded to electrodes over the hemisphere contralateral to the finger movements.

\section{D-gait analysis}

Self-reflecting markers (diameter: $14 \mathrm{~mm}$ ) were fixed bilaterally according to the Plug-in Gait model over the second metatarsal bone, the heel and lateral malleolus. Participants walked barefoot at self-selected normal speed. Gait was assessed at initiation (gait ignition) and after 3-4 m, when full walking speed was reached (steady-state gait). To avoid external cueing patients were instructed to decide by themselves to initiate walking without any start signal. 3DGA was repeated three times each before and after the TMS session. For the analysis of gait initiation, the legs were stratified according to the side, which was used to initiate locomotion (starting leg, non-starting leg), for the steady-state gait section according to the clinically more affected side by PD symptoms. Marker trajectories were recorded by eight infrared cameras (Vicon Oxford, Oxford Metrics Ltd, UK, 200Hz) analysed in Nexus 1.8.1 and were reviewed offline in Polygon 3.5.1 (Oxford Metrics, UK). The first stride upon gait ignition on each side and two strides during steady-state gait were analysed. Following parameters were provided as automatic output by Polygon: Cadence, stride length, stride time, 
step length, step time, opposite foot off, opposite foot contact, single leg support time, double limb support time (DLST) and walking speed.

\section{Transcranial magnetic stimulation}

rTMS was applied as intermittent theta burst stimulation (iTBS) bilaterally over the SMC (MagstimPro, UK) [31]. A figure-of-eight coil was held with its intersection tangentially to the skull in $45^{\circ}$ anterior-posterior orientation. The stimulation hot spot for the tibialis anterior muscle over the contralateral motor cortex, defined as the location for eliciting the largest motor evoked potential (MEP), was searched. Active and resting motor thresholds (AMT, RMT) were determined at the hot spot over both hemispheres. The respective thresholds were defined as the stimulation intensity for eliciting a MEP of $\geq 0.1 \mathrm{mV}$ in $5 / 10$ trials. Stimulation over the SMC was applied $3 \mathrm{~cm}$ rostral to the cortical representation of the contralateral tibialis anterior muscle in the same sagittal plane. Stimulation intensity was set at $100 \%$ AMT. Post-stimulation assessments were completed within 45 minutes after stimulation i.e. the expected duration of the stimulation effect [31].

\section{Statistics}

Group differences for nominal data were analyzed by the Fisher's exact test and for continuous variables by student's t-test or Mann-Whitney-U test as appropriate to normal distribution. A principal component analysis (PCA) with all gait parameters was set up to reduce the number of parameters to a few representatives. A cut-off criterion of $80 \%$ of the cumulative explained variance was used to determine the number of retained PCs. Parameters of interest were selected according to their proximity to the eigenvectors. A linear regression model was used to compare the BP amplitude between PD+FOG and PD-FOG. Each data point between $-1.0 \mathrm{sec}$ and motor onset was analyzed. To reduce the number of comparisons, the recordings were downsampled to $128 \mathrm{~Hz}$. To control further for type II errors, only epochs 
of $>12$ contiguous data points (i.e. $>90 \mathrm{msec}$ ) meeting the criterion of $\mathrm{p}<0.05$ were considered [32]. A linear mixed model with group and medication state as fixed effects and subjects as random effects was used to analyze premotor beta power. All models were corrected for total UPDRS III scores and disease duration. LED given to induce the OFF was also included as covariate. To analyze the impact of iTBS on gait performance a further linear mixed model with the gait parameter of interest as dependent, stimulation and task-repetition (i.e. unspecific pre-to-post-stimulation effect) as independent fixed effects and subjects and randomization as random effects was set up. Pearson's or Spearman's tests were used to correlate electrophysiological parameters with gait parameters. Significance level was set to $\mathrm{p}<0.05$.

\section{Results}

\section{Analysis of the gait parameters}

We reduced the list of gait parameters to three principal components explaining $81.75 \%$ of variance using PCA, namely stride length, stride time and DLST. Group comparisons revealed shorter stride length in PD+FOG at gait initiation. There was no association between the clinically more affected leg and the side of the starting leg in either group (PD+FOG: $\mathrm{p}=0.157$; PD-FOG: $\mathrm{p}=0.627$ ). During steady-state gait there was a non-significant tendency towards shorter stride length in PD+FOG on both sides (table 2)

\section{Bereitschaftspotential}

In one PD+FOG patient a severe tremor on the clinically less affected side persisted throughout the recording thus making it impossible to reliably discern voluntary muscle activity on EMG. These recordings were discarded from statistical analysis. The amplitudes of the BP over $C c$ and $C z$ prior to middle finger movements on the more affected side were 
smaller in PD+FOG in the OFF ( $C c$ : from -0.356 to $-0.188 \mathrm{sec}$, epoch length: $0.168 \mathrm{sec} ; C z$ : from -0.372 to $-0.204 \mathrm{sec}$, epoch length: $0.168 \mathrm{sec} ; \mathrm{p}<0.05)$, whereas in the $\mathrm{ON}$ the BP was comparable in both groups. Over $F c$ the BP amplitude was comparable in both groups in the OFF, but it decreased in PD+FOG before movements on the more affected side and conversely increased in PD-FOG in the ON ( $F c$ : from -0.820 to $-0.628 \mathrm{sec}$, epoch length: $0.192 \mathrm{sec} ; \mathrm{p}<0.05$ ) (figure 1). When the middle finger on the clinically less affected hand was moved, the BP amplitudes were comparable in both patient groups over all electrodes.

\section{Event-related beta desynchronisation and coherence}

There was a relative increase of beta frequency power in PD+FOG before finger movement initiation on the more affected side, while beta power remained unchanged in PD-FOG. This relative increase was observed over all electrodes with the strongest statistical effect over $C z$ i.e. the SMC. (Group effects: $C c$ : $\mathrm{F}(1,41)=8.220, \mathrm{p}=0.007 ; F c: \mathrm{F}(1,41)=6.967 \mathrm{p}=0.012 ; C z$ : $\mathrm{F}(1,41)=14.759, \mathrm{p}<0.001 ; F z: \mathrm{F}(1,41)=6.112, \mathrm{p}<0.018)$. Prior to finger movements on the less affected side, a similar increase of premotor beta power was seen in PD+FOG over $C c$ and $C z$, but not over $F c$ or $F z$ (Group effects: $C c: \mathrm{F}(1,42)=4.209, \mathrm{p}=0.046 ; C z: \mathrm{F}(1,42)=4.431$, $\mathrm{p}=0.041$ ). Medication did not have any impact on beta power over either hemisphere or electrode, respectively (for details see also supplementary material).

Premotor cortico-cortical coherence between $C c$ and $C z$, before finger movements on the more affected hand, was stronger in PD+FOG than in PD-FOG (group effect: $F(1,41)=7.444$, p=0.009) whereas it was comparable between $C c$ and $F c$ as well as between $C c$ and $F z$ in both groups and in both medication states, respectively. When the middle finger on the less affected side was extended, premotor cortico-cortical coherence between $C c$ and $C z$ was stronger in PD+FOG (group effect: $\mathrm{F}(1,42)=6.131$, $\mathrm{p}=0.018$ ), but there was no group effect for coherence between $C c$ and the other two electrodes $(F c, F z)$. Medication did not have an influence on cortico-cortical coherence either. 
Total beta power over $C z$ before finger movements on the more affected side correlated with stride length in the OFF on the starting leg $(\mathrm{r}=0.513, \mathrm{p}=0.009)$ and non-starting leg $(\mathrm{r}=0.480$, $\mathrm{p}=0.018$ ). No correlation was seen between beta power and total motor impairment as measured by the UPDRS III ( $p>0.1)$. FOG-Q scores correlated with total beta power in PD+FOG (rho=0.710, $\mathrm{p}=0.010)$, but not in PD-FOG $(\mathrm{p}>0.1)$. All correlations were done with beta power in the OFF state.

\section{Effect of intermittent theta burst stimulation}

There were no differences regarding AMT and RMT between the visits with sham stimulation and those with iTBS $(\mathrm{P}>0.5$; see supplementary material). Overall stride time at gait initiation decreased after both sham stimulation and iTBS (task repetition effect: starting leg: $\mathrm{F}(1,129)=18.937, \mathrm{p}<0.001$; non-starting leg: $\mathrm{F}(1,129)=7.074, \mathrm{p}=0.009)$. After iTBS, this decrease of stride time was less prominent on the starting leg (stimulation $\times$ task repetition: $(\mathrm{F}(1,129)=4.190, \mathrm{p}=0.043)$ and almost absent on the non-starting leg (stimulation $\times$ task repetition: $\mathrm{F}(1,129)=4.920, \mathrm{p}=0.028)$. Neither of the stimulation modes had an effect on stride time during steady-state gait.

At gait initiation there was a trend towards increased stride length on the non-starting leg after sham, whereas stride length remained widely unchanged after iTBS (stimulation $\times$ task repetition: $\mathrm{F}(1,129)=3.606 \mathrm{p}=0.060)$. No stimulation effect was seen on the starting leg. Stride length increased during steady-state gait on both legs after sham and iTBS (task repetition effect : dominant leg: $\mathrm{F}(1,129)=9.904, \mathrm{p}=0.002$; non-dominant leg: $\mathrm{F}(1,129)=9.774, \mathrm{p}=0.002)$. There was a trend towards a smaller increase of stride length on both legs after iTBS compared to sham (stimulation $\times$ task repetition: dominant leg: $\mathrm{F}(1,129)=2.769, \mathrm{p}=0.099)$; non-dominant leg: $\mathrm{F}(1,129)=3.030, \mathrm{p}=0.084)$. Neither task repetition (i.e. pre-to-post effect) nor the stimulation mode had an impact on double leg support time. 


\section{Discussion}

We performed a multimodal analysis of the contribution of SMC activation to FOG in PD. Firstly, we saw quantitative difference in the activation of the SMC, but also of the M1 between PD+FOG and PD-FOG, as reflected by reduced amplitudes of the BP over $C z$ and $C c$, respectively, in PD+FOG. Secondly, we observed qualitative alterations of neuronal activity within the SMC consisting of a 'paradoxical' increase of beta activity along with increased cortico-cortical coherence between the SMC and M1 prior to voluntary movements in PD+FOG. Thirdly, beta activity from the SMC correlated with FOG-related kinematic measures, namely stride length at gait initiation and, in the group of PD+FOG, with FOG severity. Since these findings were much more prominent over the hemisphere contralateral to the more affected side, we posit that the observed group differences are linked to neurodegeneration within the dopaminergic system [33]. Our findings are also in line with the observation of FOG being more likely to occur on the clinically more affected side [34]. The groups were widely comparable with regard to clinical characteristics, in particular motor impairment, and all findings were significant after correcting for UPDRS III and disease duration. Therefore we believe that the observed statistical effects are specific for the presence of FOG and may not be ascribed to the impact of global motor impairment or disease progression alone.

In the interventional part of our study we observed a relative worsening of gait performance, particularly of stride time, in PD+FOG at gait initiation, after modulating SMC neuronal activity by a facilitating rTMS paradigm. This finding supports the notion of a greater engagement of the SMC at gait initiation than during automatized gait, the latter being mainly maintained by basal ganglia-brainstem loops $[35,36]$. Together with the EEG results, the rTMS findings point to a central role of the SMC with regard to the movement ignition failure, commonly seen in PD patients with FOG. These findings, however, contrast our initial 
hypothesis that iTBS may have a beneficial effect on interepisodical gait performance in PD+FOG.

\section{Bereitschaftspotential and FOG}

We included the BP as surrogate marker for preparatory processes within the SMC prior to uncued movements $[10,37]$. To record the BP (and also premotor beta activity), we did not use gait initiation itself, but the paradigm of selective self-guided, uncued finger-extensions, which was, compared to walking or stepping tasks, technically more feasible and less prone to technical artifacts [38]. It should be noted that the BP depends less on the limb used rather than on the complexity of the task [10]. A further argument was that freezing is not restricted to gait, but is a more general PD-related phenomenon within the motor system [7]. The reduced $\mathrm{BP}$ over the SMC and the M1 likely reflects impaired SMC activation during volitional movements including impaired propagation of the neuronal signal to M1. The notion of disturbed connectivity between SMC and M1 in PD is corroborated by data from an fMRI study [39]. According to general electrophysiological principles the amplitude of motor potentials mainly depends on the number of excited neurons [40]. In line with this argumentation reduced BP amplitudes indicate impaired recruitment or decreased availability of excitable neurons within the SMC at motor initiation in PD+FOG.

Previous studies consistently showed attenuated BP amplitudes in PD in general, particularly for the early component, which was assumed to reflect decreased mesial premotor cortex activation during motor preparation [11, 37, 41, 42]. In our study the BP amplitudes differed between PD+FOG and PD-FOG only in the OFF thus suggesting dopamine-dependent group differences in SMC activation. This observation is in line with previous studies that demonstrated a certain degree of normalization of the BP after the intake of dopaminergics, although the effect of dopamine on the BP was only investigated in PD patients regardless of 
the presence of FOG $[43,44]$ Our findings are also congruent with an absent BP in patients with strokes involving the SMC who may even present with FOG-like gait patterns [12].

Beyond this there was a relative amplitude decrease over the lateral premotor cortex in $\mathrm{PD}+\mathrm{FOG}$ in the $\mathrm{ON}$, which again was only seen over the hemisphere contralateral to the more affected body side. The degree of neuronal activity within this network, however, does not exceed that seen in PD-FOG. We interpret these findings as a compensatory recruitment of the lateral premotor cortex in PD+FOG in the OFF. There is a considerable body of evidence demonstrating that PD patients, particularly in the state OFF medication, rely more on lateral premotor networks. These networks are predominantly involved in cued movements and are presumably better preserved than those for uncued movements [37, 41, 45]. It has furthermore been shown that the amplitude of BP is increased by sensory feedback [46]. Hence a larger BP amplitude over lateral electrodes in the OFF, as seen in our study in PD+FOG, could reflect reinforced sensorimotor integration in this group, particularly in the hypodopaminergic state.

\section{Beta oscillations and FOG}

Beta oscillations are considered as an idling rhythm in the motor system that preserves the current motor state and that needs to be desynchronized when a motor program is changed voluntarily [22]. To date the role of pathological beta with regard to FOG is supported by a few studies, which demonstrated increased beta, particularly within the STN, in PD+FOG [47-49], whereas the role of cortical beta oscillations still remains elusive [50]. In addition to these previous findings, we observed a 'paradoxical' increase of beta activity in PD+FOG, but not in PD-FOG before volitional movements. However, clear beta desynchronisation was not seen in PD-FOG either. Our findings are congruent with previous studies that demonstrated disturbed beta desynchronisation in PD [25]. When the clinically more affected side was moved, increased beta oscillations in PD+FOG were found over all electrodes, although the 
statistical effect was strongest over $\mathrm{Cz}$ i.e. the SMC. On the other hand increased beta power was only found over the SMC and the M1, but not over the lateral premotor cortex, when finger movements on the less affected side were initiated. We believe that the same considerations regarding the relationship of our findings with neurodegeneration in the dopaminergic system, as discussed above for the BP, apply for pathological beta oscillations. Furthermore, connectivity between SMC and M1 as reflected by cortico-cortical coherence in the beta range was significantly stronger in PD+FOG over both hemispheres. In contrast to comparable approaches in PD patients [24, 51-53], we did not find restored beta desynchronisation after the intake of dopaminergics in either group. The reasons for this difference, however, remain unclear.

Taken together, our study revealed increased beta activity, and oscillatory synchrony predominantly in the network that is engaged in uncued movements and that encompasses the SMC and M1. Increased synchrony in the beta band may render the network less responsive for input signals thus reducing its information coding capacity [54]. In our study we did not investigate beta activity directly during FOG episodes. But it should be noted that our findings of increased premotor beta correlated with gait parameters at gait initiation and FOG severity. These findings, however, do not provide holistic explanation for FOG itself, but they should be regarded more as a prerequisite, which increase the probability for the occurrence of an FOG episode.

\section{Detrimental effect of SMC-iTBS on gait performance}

We used rTMS in a randomized cross-over manner to modulate SMC activity and to elucidate the cause-consequence relationship of SMC alterations with FOG. To investigate the impact of SMC-iTBS we focused on FOG-related interepisodical changes of gait rather than on actual FOG episodes. Interepisodical spatiotemporal gait parameters are more robust, whereas FOG itself is less reproducible under experimental conditions [55]. After the stimulation 
block we observed an increase of stride length along with a decrease of stride time, whereas DLST remained unchanged. We considered the combination of these findings as an improvement of gait after stimulation. After real iTBS, however, this post-stimulation effect was attenuated for stride time and, although less strikingly, for stride length. The statistical effect of iTBS on the gait parameters was more prominent at gait initiation than during steady-state gait (i.e. during automatized maintained gait). We propose that repeating the task may have led to a certain motor sequence learning effect, particularly in the cognitively more demanding gait initiation paradigm. iTBS may then have negatively interfered with these learning processes. Which domain exactly was responsible for the learning effect remains speculative, because we did not perform a sophisticated cognitive assessment before and after rTMS. Arguably it could have been mediated within the visuospatial domain, which among other hubs strongly engages the SMC [56].

Repetitive TMS over the premotor cortex for FOG was already investigated in previous studies: Kim et al. showed an improvement of FOG and gait after facilitating rTMS over the SMC [57]. Conversely Tard et al. found no benefit of a single session iTBS over the premotor cortex. Different to our study, patients were investigated in the ON in these two studies. There were furthermore methodological differences in localizing the hotspot. Another study did not show any benefit from excitatory rTMS over the SMC [58]. The latter authors, however, also included patients with atypical Parkinsonism. Low-frequency (i.e. inhibitory) rTMS over the SMC was applied in one study and was found to mildly improve gait as measured by the UPDRS gait item [59]. Overall excitatory rTMS paradigms applied over the SMC seem to be less beneficial with regard to FOG and gait improvement in PD than inhibitory paradigms. One possible explanation might be that facilitating paradigms reinforce pathological networks between the SMC and connected hubs. 


\section{Conceptual considerations}

According to current concepts of motor control the SMC is embedded in a network, which mediates predominantly self-guided, uncued movements. Conversely, cued movements are organized through more lateral networks such as the lateral prefrontal cortex [37]. In PD neuronal circuits for self-guided movements are disproportionately stronger disrupted whereas those for cued movements are better preserved, and they may even be engaged to overcome motor impairment e.g. by cueing strategies in FOG [37, 60]. Our findings of more severe quantitative and qualitative alterations of neuronal activity over the SMC and M1 in PD+FOG fit quite well into this concept. The findings over lateral network nodes, however, were more alike in both groups thus being in line with the argumentation that these network are generally less severely affected in PD [37, 41] and in FOG in particular [61]. We propose that in PD+FOG there is increased beta oscillatory activity in the more deficient network for selfguided uncued movement encompassing the SMC and M1. Excitatory rTMS over the SMC may have even further reinforced these pathological oscillations. This reinforced SMC connectivity fosters the current motor state, reduces the coding capacity of the motor network and thus hinders accurate beta desynchronisation, required for shifting the motor set at movement initiation [23]. Furthermore rTMS may have shifted motor control away from a compensatory reliance on external cues, mainly mediated through lateral network hubs, towards the network for uncued movements. The few study available to date including ours propose that increased beta activity is certainly a relevant electrophysiological trait in PD+FOG [48, 49], although its exact role in the pathophysiology of FOG needs to be further established.

\section{Conclusion}

In this study we demonstrated that there were qualitative and quantitative alterations of SMC functioning in PD patients with FOG compared to those without. Reduced activation of the 
SMC along with increased SMC connectivity in the beta frequency range hinder a flexible shift of the motor set as it is required for gait initiation. However, further studies are warranted to elucidate how beta activity exactly drives the occurrence of a single FOG episode. With regard to clinical relevance increased knowledge on FOG-related beta activity moreover may support the development of adaptive deep brain stimulation systems in PD (including EEG-based closed loop systems), which are rapidly arising on the horizon [62, 63]. 


\section{$\underline{\text { Legends }}$}

Figure 1: The Bereitschaftspotential curves recorded over $C c, C z, F c$ and $F z$ are shown. The plots on the left side include the BP following middle finger extensions on the clinically more affected hand in the OFF, those on the right side the BP following middle finger movements in the ON. The grand average BP from PD+FOG is shown as solid line, the grand average BP from PD-FOG as dashed line. The horizontal bars (placed at $3 \mu \mathrm{V}$ ) indicate epochs when the group comparison met the criterion of $\mathrm{p}<0.05$ for $>12$ subsequent data points. The two vertical lines show the borders of the time epoch, which was statistically analyzed. The time values are provided as latencies in relation to motor onset $(\mathrm{t}=0 \mathrm{sec}$.)

Figure 2: The results for beta desynchronisation related to middle finger extensions on the clinically more affected side are shown (mean $+/-95 \% \mathrm{CI})$. The dark grey bars indicate the results in the OFF, those in bright grey the results in the ON. According to the formula applied for the calculation of beta desynchronisation (for details see the methods section), negative values indicate an increase in beta power before motor onset. The results for the movements on the less affected hand are provided in the supplementary materials

Figure 3:

The iTBS data for stride time (seconds) and stride length (meters) at gait initiation are shown. The black line indicates the data for the session with iTBS, the bright grey lines the data for the sham session. The asterisks $(*)$ indicate significant stimulation $\times$ task repetition interactions $(\mathrm{p}<0.05)$, the diamond $(\#)$ those results which were borderline significant $(\mathrm{p}<0.1)$. Abbreviations: iTBS - intermittent theta burst stimulation 
Table 1: Baseline characteristics

\begin{tabular}{|c|c|c|c|}
\hline & $\begin{array}{c}\text { PD+FOG } \\
(\mathrm{N}=12)\end{array}$ & $\begin{array}{c}\text { PD-FOG } \\
(\mathrm{N}=11)\end{array}$ & $\begin{array}{c}\text { Significance } \\
\text { (p-value) }\end{array}$ \\
\hline Age & $\begin{array}{c}61.9 \pm 8.5 \\
64.30(15.4)\end{array}$ & $\begin{array}{c}61.05 .7 \\
63.0(8.8)\end{array}$ & 0.79 \\
\hline $\operatorname{Sex}(m / f)$ & $10 / 2$ & $10 / 1$ & 1.00 \\
\hline Disease duration & $\begin{array}{l}12.7 \pm 4.3 \\
12.5(4.8)\end{array}$ & $\begin{array}{l}7.4 \pm 3.5 \\
6.0 \pm 4.0\end{array}$ & $<0.01$ \\
\hline Hoehn\&Yahr & $\begin{array}{l}2.3 \pm 0.4 \\
2.0(0.9)\end{array}$ & $\begin{array}{l}2.1 \pm 0.2 \\
2.0 \pm 0.0\end{array}$ & 0.17 \\
\hline UPDRS I & $\begin{array}{l}2.4 \pm 1.6 \\
3.0(3.5)\end{array}$ & $\begin{array}{l}1.9 \pm 1.6 \\
2.0(2.0)\end{array}$ & 0.41 \\
\hline UPDRS II & $\begin{array}{c}16.0 \pm 5.6 \\
16.50(10.50)\end{array}$ & $\begin{array}{l}8.9 \pm 2.9 \\
10.0(4.0)\end{array}$ & $<0.01$ \\
\hline $\begin{array}{l}\text { UPDRS III (OFF) } \\
\text { (blinded) }\end{array}$ & $\begin{array}{l}28.5 \pm 7.7 \\
29.8(8.1)\end{array}$ & $\begin{array}{l}23.2 \pm 6.7 \\
24.0(12.0)\end{array}$ & 0.12 \\
\hline $\begin{array}{l}\text { UPDRS III (OFF) } \\
\text { (blinded, incl. } \\
\text { rigidigy item) }\end{array}$ & $\begin{array}{c}33.87 .9 \\
37.3(9.4)\end{array}$ & $\begin{array}{l}27.3 \pm 7.7 \\
28.0(12.0)\end{array}$ & 0.06 \\
\hline FOG-Q & $\begin{array}{l}10.9 \pm 3.5 \\
10.5(1.8)\end{array}$ & $\begin{array}{l}2.3 \pm 1.7 \\
2.0(2.0)\end{array}$ & $<0.01$ \\
\hline FOG duration & $\begin{array}{l}4.6 \pm 4.3 \\
3.0(7.0)\end{array}$ & n.a. & n.a. \\
\hline FAB & $\begin{array}{l}14.8 \pm 2.2 \\
15.0(3.5)\end{array}$ & $\begin{array}{l}16.2 \pm 1.1 \\
16.0(1.0)\end{array}$ & 0.10 \\
\hline MMS & $\begin{array}{l}28.7 \pm 1.3 \\
29.0(2.0)\end{array}$ & $\begin{array}{l}28.8 \pm 1.2 \\
29.0(2.0)\end{array}$ & 0.77 \\
\hline LED & $\begin{array}{l}1334.0 \pm 636.4 \\
1269.1(602.5)\end{array}$ & $\begin{array}{l}905.2 \pm 303.2 \\
949.0(542.4)\end{array}$ & 0.06 \\
\hline
\end{tabular}

Results are reported as means \pm standard deviation as well as medians (interquartile range). Abbreviations: FAB - Frontal Assessment Battery, FOG - Freezing of Gait, FOG-Q - Freezing of Gait Questionnaire, LED - Levodopa Equivalence Dose, MMSE - Mini Mental Status Examination, PD+FOG - Parkinson's disease patients with freezing of gait, PD-FOG Parkinson's disease patients without freezing of gait, UPDRS - Unified Parkinson's Disease Rating Scale. 
Table 2: Group comparison of gait parameters

\begin{tabular}{|c|c|c|c|}
\hline & PD+FOG & PD-FOG & p-value \\
\hline \multicolumn{4}{|c|}{ gait initiation } \\
\hline \multicolumn{4}{|c|}{ starting leg } \\
\hline Stride length & $1.05 \pm 0.18$ & $1.26 \pm 0.15$ & $<0.01$ \\
\hline Stride time & $1.11 \pm 0.11$ & $1.12 \pm 0.10$ & 0.68 \\
\hline DLST (\%) & $49.81 \pm 6.08$ & $46.28 \pm 4.37$ & 0.13 \\
\hline \multicolumn{4}{|c|}{ non-starting leg } \\
\hline Stride length & $1.02 \pm 0.18$ & $1.24 \pm 0.15$ & $<0.01$ \\
\hline Stride time & $1.23 \pm 0.15$ & $1.24 \pm 0.14$ & 0.80 \\
\hline DLST (\%) & $52.67 \pm 7.13$ & $51.09 \pm 4.36$ & 0.31 \\
\hline \multicolumn{4}{|c|}{ steady-state gait } \\
\hline \multicolumn{4}{|c|}{ more affected leg } \\
\hline Stride length & $1.13 \pm 0.18$ & $1.26 \pm 0.15$ & 0.09 \\
\hline Stride time & $1.01 \pm 0.06$ & $1.05 \pm 0.08$ & 0.17 \\
\hline DLST (\%) & $46.31 \pm 5.18$ & $45.92 \pm 4.14$ & 0.85 \\
\hline \multicolumn{4}{|c|}{ less affected leg } \\
\hline Stride length & $1.13 \pm 0.18$ & $1.26 \pm 0.15$ & 0.08 \\
\hline Stride time & $1.01 \pm 0.06$ & $1.05 \pm 0.08$ & 0.18 \\
\hline DLST (\%) & $45.93 \pm 4.64$ & $45.09 \pm 3.87$ & 0.64 \\
\hline
\end{tabular}

Results are reported as means \pm standard deviation. Abbreviations: DLST - double leg support time, PD+FOG - Parkinson's disease patients with freezing of gait, PD-FOG - Parkinson's disease patients without freezing of gait. 
Figure 1: Bereitschaftspotential results
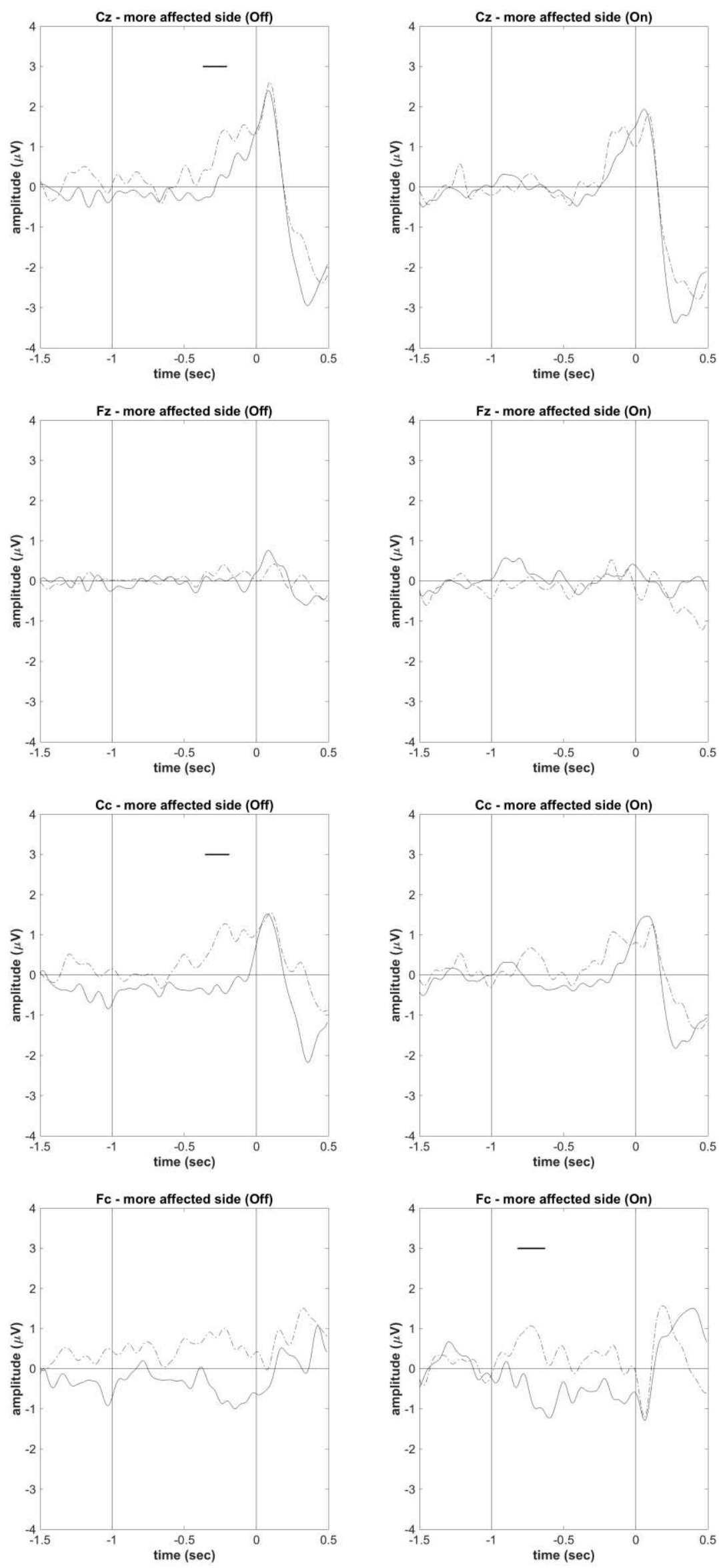
Figure 2: Beta desynchronisation
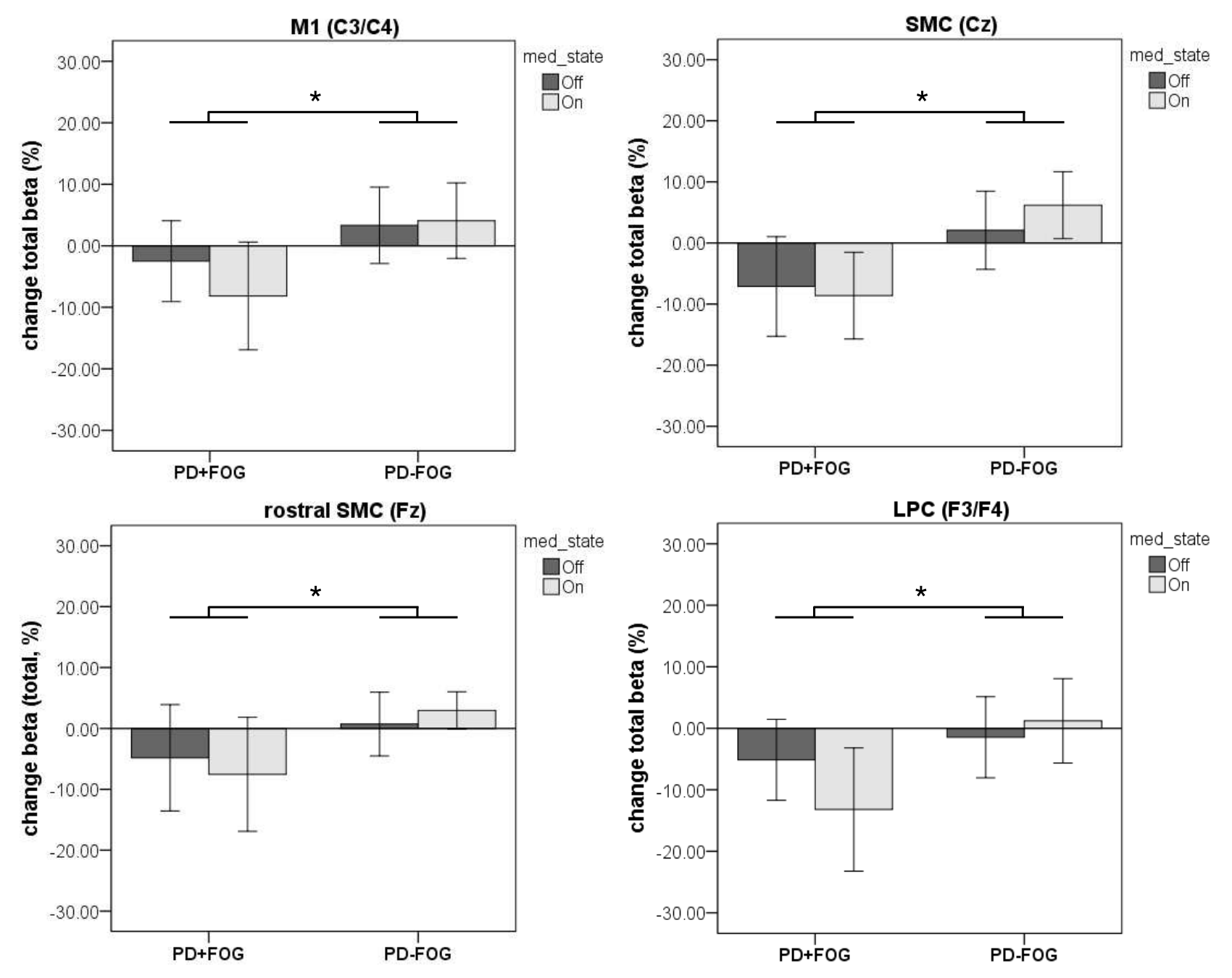
Figure 3: iTBS results at gait initiation
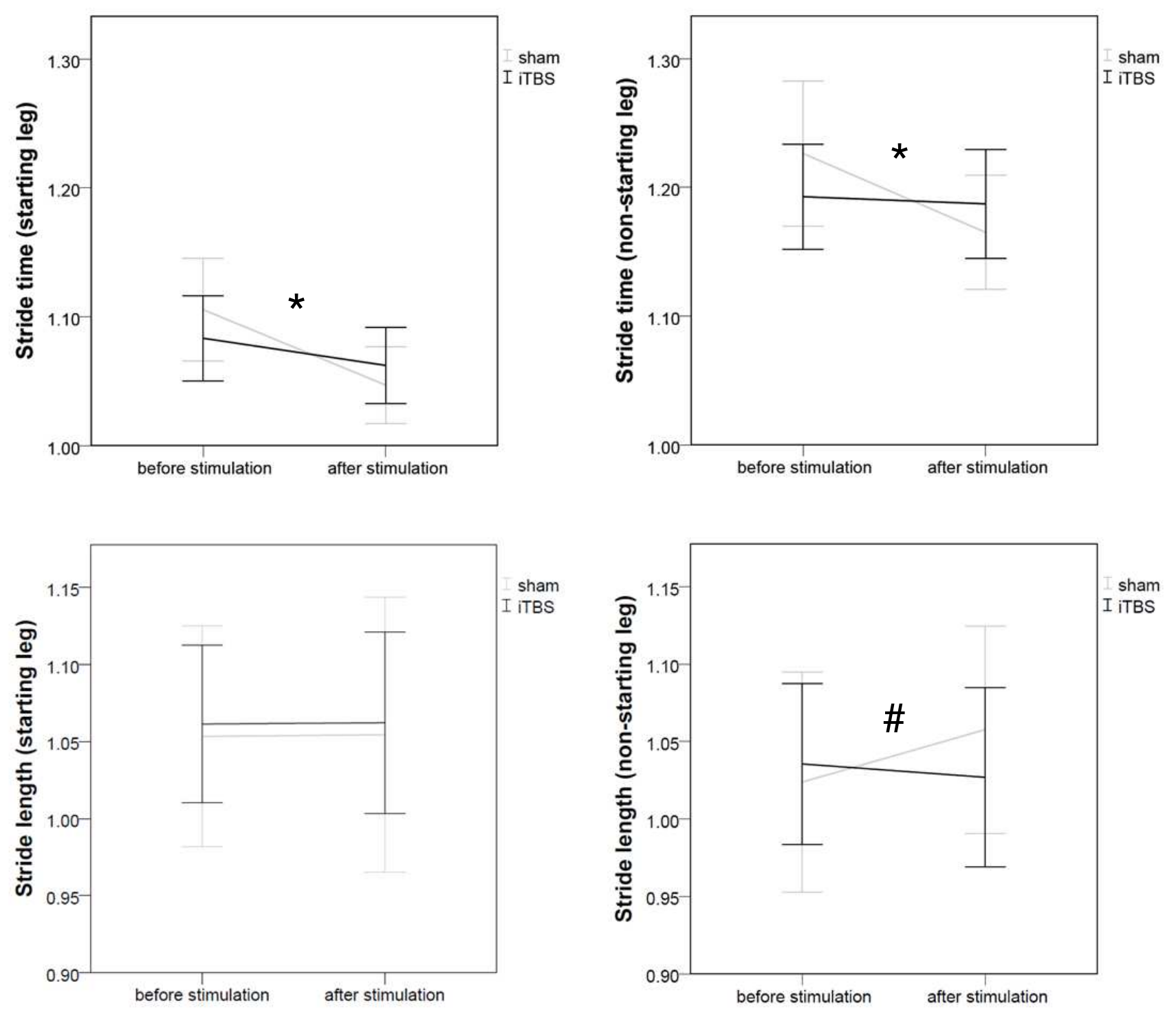


\section{References}

[1] J.G. Nutt, B.R. Bloem, N. Giladi, M. Hallett, F.B. Horak, A. Nieuwboer, Freezing of gait: moving forward on a mysterious clinical phenomenon, The Lancet. Neurology 10(8) (2011) 734-44.

[2] Y. Okuma, N. Yanagisawa, The clinical spectrum of freezing of gait in Parkinson's disease, Movement disorders : official journal of the Movement Disorder Society 23 Suppl 2 (2008) S426-30.

[3] A.J. Espay, A. Fasano, B.F. van Nuenen, M.M. Payne, A.H. Snijders, B.R. Bloem, "On" state freezing of gait in Parkinson disease: a paradoxical levodopa-induced complication, Neurology 78(7) (2012) 454-7.

[4] Y. Okada, T. Fukumoto, K. Takatori, K. Nagino, K. Hiraoka, Variable initial swing side and prolonged double limb support represent abnormalities of the first three steps of gait initiation in patients with Parkinson's disease with freezing of gait, Frontiers in neurology 2 (2011) 85.

[5] Y. Okada, T. Fukumoto, K. Takatori, K. Nagino, K. Hiraoka, Abnormalities of the first three steps of gait initiation in patients with Parkinson's disease with freezing of gait, Parkinson's disease 2011 (2011) 202937.

[6] F. Brugger, S. Jesus, C. Ganos, K.P. Bhatia, Episodic akinesia of jaw movements akin to freezing in multiple system atrophy, Parkinsonism \& related disorders 21(12) (2015) 1483-4.

[7] S. Vercruysse, J. Spildooren, E. Heremans, N. Wenderoth, S.P. Swinnen, W. Vandenberghe, A. Nieuwboer, The neural correlates of upper limb motor blocks in Parkinson's disease and their relation to freezing of gait, Cerebral cortex 24(12) (2014) 3154-66.

[8] S.J. Lewis, J.M. Shine, The Next Step: A Common Neural Mechanism for Freezing of Gait, The Neuroscientist : a review journal bringing neurobiology, neurology and psychiatry 22(1) (2016) 72-82.

[9] P. Nachev, C. Kennard, M. Husain, Functional role of the supplementary and pre-supplementary motor areas, Nature reviews. Neuroscience 9(11) (2008) 856-69.

[10] H. Shibasaki, M. Hallett, What is the Bereitschaftspotential?, Clinical neurophysiology : official journal of the International Federation of Clinical Neurophysiology 117(11) (2006) 2341-56.

[11] D. Georgiev, F. Lange, C. Seer, B. Kopp, M. Jahanshahi, Movement-related potentials in Parkinson's disease, Clinical neurophysiology : official journal of the International Federation of Clinical Neurophysiology 127(6) (2016) 2509-19.

[12] F. Brugger, M. Galovic, B.J. Weder, G. Kagi, Supplementary Motor Complex and Disturbed Motor Control - a Retrospective Clinical and Lesion Analysis of Patients after Anterior Cerebral Artery Stroke, Frontiers in neurology 6 (2015) 209.

[13] S. Della Sala, A. Francescani, H. Spinnler, Gait apraxia after bilateral supplementary motor area lesion, Journal of neurology, neurosurgery, and psychiatry 72(1) (2002) 77-85.

[14] H.W. Hwang, S.H. Lee, C.H. Lyoo, M.S. Lee, Paroxysmal freezing of gait in a patient with mesial frontal transient ischemic attacks, BMC neurology 17(1) (2017) 122.

[15] A.H. Snijders, I. Leunissen, M. Bakker, S. Overeem, R.C. Helmich, B.R. Bloem, I. Toni, Gait-related cerebral alterations in patients with Parkinson's disease with freezing of gait, Brain : a journal of neurology 134(Pt 1) (2011) 59-72.

[16] T. Wu, J. Zhang, M. Hallett, T. Feng, Y. Hou, P. Chan, Neural correlates underlying micrographia in Parkinson's disease, Brain : a journal of neurology 139(Pt 1) (2016) 144-60.

[17] J.M. Shine, E. Matar, P.B. Ward, M.J. Frank, A.A. Moustafa, M. Pearson, S.L. Naismith, S.J. Lewis, Freezing of gait in Parkinson's disease is associated with functional decoupling between the cognitive control network and the basal ganglia, Brain : a journal of neurology 136(Pt 12) (2013) 3671-81.

[18] J.M. Shine, E. Matar, P.B. Ward, S.J. Bolitho, M. Pearson, S.L. Naismith, S.J. Lewis, Differential neural activation patterns in patients with Parkinson's disease and freezing of gait in response to concurrent cognitive and motor load, PloS one 8(1) (2013) e52602.

[19] D.S. Peterson, K.A. Pickett, R. Duncan, J. Perlmutter, G.M. Earhart, Gait-related brain activity in people with Parkinson disease with freezing of gait, PloS one 9(3) (2014) e90634.

[20] B.W. Fling, R.G. Cohen, M. Mancini, S.D. Carpenter, D.A. Fair, J.G. Nutt, F.B. Horak, Functional reorganization of the locomotor network in Parkinson patients with freezing of gait, PloS one 9(6) (2014) e100291. 
[21] J.M. Hall, J.M. Shine, K.A. Ehgoetz Martens, M. Gilat, K.M. Broadhouse, J.Y.Y. Szeto, C.C. Walton, A.A. Moustafa, S.J.G. Lewis, Alterations in white matter network topology contribute to freezing of gait in Parkinson's disease, Journal of neurology 265(6) (2018) 1353-1364.

[22] P. Brown, Oscillatory nature of human basal ganglia activity: relationship to the pathophysiology of Parkinson's disease, Movement disorders : official journal of the Movement Disorder Society 18(4) (2003) 357-63.

[23] J.S. Brittain, P. Brown, Oscillations and the basal ganglia: motor control and beyond, Neurolmage $85 \mathrm{Pt}$ 2 (2014) 637-47.

[24] P. Brown, C.D. Marsden, Bradykinesia and impairment of EEG desynchronization in Parkinson's disease, Movement disorders : official journal of the Movement Disorder Society 14(3) (1999) 423-9.

[25] H.C. Wang, A.J. Lees, P. Brown, Impairment of EEG desynchronisation before and during movement and its relation to bradykinesia in Parkinson's disease, Journal of neurology, neurosurgery, and psychiatry 66(4) (1999) 442-6.

[26] M. Scholten, R.B. Govindan, C. Braun, B.R. Bloem, C. Plewnia, R. Kruger, A. Gharabaghi, D. Weiss, Cortical correlates of susceptibility to upper limb freezing in Parkinson's disease, Clinical neurophysiology : official journal of the International Federation of Clinical Neurophysiology 127(6) (2016) 2386-93.

[27] N. Giladi, H. Shabtai, E.S. Simon, S. Biran, J. Tal, A.D. Korczyn, Construction of freezing of gait questionnaire for patients with Parkinsonism, Parkinsonism \& related disorders 6(3) (2000) 165-170.

[28] R.W. Homan, J. Herman, P. Purdy, Cerebral location of international 10-20 system electrode placement, Electroencephalography and clinical neurophysiology 66(4) (1987) 376-82.

[29] H. Steinmetz, G. Furst, B.U. Meyer, Craniocerebral topography within the international 10-20 system, Electroencephalography and clinical neurophysiology 72(6) (1989) 499-506.

[30] D.M. Halliday, J.R. Rosenberg, A.M. Amjad, P. Breeze, B.A. Conway, S.F. Farmer, A framework for the analysis of mixed time series/point process data--theory and application to the study of physiological tremor, single motor unit discharges and electromyograms, Progress in biophysics and molecular biology 64(2-3) (1995) 237-78.

[31] Y.Z. Huang, M.J. Edwards, E. Rounis, K.P. Bhatia, J.C. Rothwell, Theta burst stimulation of the human motor cortex, Neuron 45(2) (2005) 201-6.

[32] D. Guthrie, J.S. Buchwald, Significance testing of difference potentials, Psychophysiology 28(2) (1991) 240-4.

[33] F. Ba, W.R. Martin, Dopamine transporter imaging as a diagnostic tool for parkinsonism and related disorders in clinical practice, Parkinsonism \& related disorders 21(2) (2015) 87-94.

[34] A. Nieuwboer, F. Chavret, A. Willems, K. Desloovere, Does freezing in Parkinson's disease change limb coordination? A kinematic analysis, Journal of neurology 254(9) (2007) 1268-77.

[35] K. Takakusaki, Forebrain control of locomotor behaviors, Brain research reviews 57(1) (2008) 192-8.

[36] K. Takakusaki, Neurophysiology of gait: from the spinal cord to the frontal lobe, Movement disorders : official journal of the Movement Disorder Society 28(11) (2013) 1483-91.

[37] M. Jahanshahi, I.H. Jenkins, R.G. Brown, C.D. Marsden, R.E. Passingham, D.J. Brooks, Self-initiated versus externally triggered movements. I. An investigation using measurement of regional cerebral blood flow with PET and movement-related potentials in normal and Parkinson's disease subjects, Brain : a journal of neurology 118 ( Pt 4) (1995) 913-33.

[38] M. Vidailhet, P.R. Atchison, F. Stocchi, P.D. Thompson, J.C. Rothwell, C.D. Marsden, The bereitschaftspotential preceding stepping in patients with isolated gait ignition failure, Movement disorders : official journal of the Movement Disorder Society 10(1) (1995) 18-21.

[39] E. Canu, F. Agosta, E. Sarasso, M.A. Volonte, S. Basaia, T. Stojkovic, E. Stefanova, G. Comi, A. Falini, V.S. Kostic, R. Gatti, M. Filippi, Brain structural and functional connectivity in Parkinson's disease with freezing of gait, Human brain mapping 36(12) (2015) 5064-78.

[40] P.L. Nunez, R. Srinavasan, Electric Fields of the Brain: The Neurophysics of EEG., Oxford University Press, Oxford, UK, 2006.

[41] R. Cunnington, R. lansek, J.L. Bradshaw, J.G. Phillips, Movement-related potentials in Parkinson's disease. Presence and predictability of temporal and spatial cues, Brain : a journal of neurology 118 ( Pt 4) (1995) 935-50. 
[42] J.P. Dick, J.C. Rothwell, B.L. Day, R. Cantello, O. Buruma, M. Gioux, R. Benecke, A. Berardelli, P.D. Thompson, C.D. Marsden, The Bereitschaftspotential is abnormal in Parkinson's disease, Brain : a journal of neurology 112 ( Pt 1) (1989) 233-44.

[43] J.P. Dick, R. Cantello, O. Buruma, M. Gioux, R. Benecke, B.L. Day, J.C. Rothwell, P.D. Thompson, C.D. Marsden, The Bereitschaftspotential, L-DOPA and Parkinson's disease, Electroencephalography and clinical neurophysiology 66(3) (1987) 263-74.

[44] A.P. Feve, N. Bathien, P. Rondot, Chronic administration of L-dopa affects the movement-related cortical potentials of patients with Parkinson's disease, Clinical neuropharmacology 15(2) (1992) 100-8.

[45] J.S. Butler, C. Fearon, I. Killane, S.M. Waechter, R.B. Reilly, T. Lynch, Motor preparation rather than decision-making differentiates Parkinson's disease patients with and without freezing of gait, Clinical neurophysiology : official journal of the International Federation of Clinical Neurophysiology 128(3) (2017) 463-471.

[46] D. Reznik, S. Simon, R. Mukamel, Predicted sensory consequences of voluntary actions modulate amplitude of preceding readiness potentials, Neuropsychologia 119 (2018) 302-307.

[47] A.P. Singh, A.; Krammermeier, S.; Mehrkens, JH.; Ilmberger, J.;Bötzel, K., Freezing of gait-related oscillatory activity in the human subthalamic nucleus, Basal Ganglia 3(1) (2013) 25-32.

[48] L. Storzer, M. Butz, J. Hirschmann, O. Abbasi, M. Gratkowski, D. Saupe, J. Vesper, S.S. Dalal, A. Schnitzler, Bicycling suppresses abnormal beta synchrony in the Parkinsonian basal ganglia, Annals of neurology 82(4) (2017) 592-601.

[49] J.B. Toledo, J. Lopez-Azcarate, D. Garcia-Garcia, J. Guridi, M. Valencia, J. Artieda, J. Obeso, M. Alegre, M. Rodriguez-Oroz, High beta activity in the subthalamic nucleus and freezing of gait in Parkinson's disease, Neurobiology of disease 64 (2014) 60-5.

[50] J. Arens, L. Storzer, J. Hirschmann, S.S. Dalal, A. Schnitzler, M. Butz, Freezing of gait does not modulate beta oscillations in mesial cortical motor areas, Movement disorders : official journal of the Movement Disorder Society 34(3) (2019) 436.

[51] A.A. Kuhn, F. Kempf, C. Brucke, L. Gaynor Doyle, I. Martinez-Torres, A. Pogosyan, T. Trottenberg, A. Kupsch, G.H. Schneider, M.I. Hariz, W. Vandenberghe, B. Nuttin, P. Brown, High-frequency stimulation of the subthalamic nucleus suppresses oscillatory beta activity in patients with Parkinson's disease in parallel with improvement in motor performance, The Journal of neuroscience : the official journal of the Society for Neuroscience 28(24) (2008) 6165-73.

[52] A.A. Kuhn, A. Kupsch, G.H. Schneider, P. Brown, Reduction in subthalamic 8-35 Hz oscillatory activity correlates with clinical improvement in Parkinson's disease, The European journal of neuroscience 23(7) (2006) 1956-60.

[53] N.J. Ray, N. Jenkinson, S. Wang, P. Holland, J.S. Brittain, C. Joint, J.F. Stein, T. Aziz, Local field potential beta activity in the subthalamic nucleus of patients with Parkinson's disease is associated with improvements in bradykinesia after dopamine and deep brain stimulation, Experimental neurology 213(1) (2008) 108-13.

[54] S. Little, P. Brown, The functional role of beta oscillations in Parkinson's disease, Parkinsonism \& related disorders 20 Suppl 1 (2014) S44-8.

[55] A.H. Snijders, M.J. Nijkrake, M. Bakker, M. Munneke, C. Wind, B.R. Bloem, Clinimetrics of freezing of gait, Movement disorders : official journal of the Movement Disorder Society 23 Suppl 2 (2008) S468-74.

[56] K. Sakai, O. Hikosaka, S. Miyauchi, Y. Sasaki, N. Fujimaki, B. Putz, Presupplementary motor area activation during sequence learning reflects visuo-motor association, The Journal of neuroscience : the official journal of the Society for Neuroscience 19(10) (1999) RC1.

[57] S.J. Kim, S.H. Paeng, S.Y. Kang, Stimulation in Supplementary Motor Area Versus Motor Cortex for Freezing of Gait in Parkinson's Disease, Journal of clinical neurology 14(3) (2018) 320-326.

[58] S.Y. Lee, M.S. Kim, W.H. Chang, J.W. Cho, J.Y. Youn, Y.H. Kim, Effects of repetitive transcranial magnetic stimulation on freezing of gait in patients with Parkinsonism, Restorative neurology and neuroscience 32(6) (2014) 743-53.

[59] Y. Shirota, H. Ohtsu, M. Hamada, H. Enomoto, Y. Ugawa, T.M.S.T.o.P.s.D. Research Committee on r, Supplementary motor area stimulation for Parkinson disease: a randomized controlled study, Neurology 80(15) (2013) 1400-5. 
[60] J. Michely, L.J. Volz, M.T. Barbe, F. Hoffstaedter, S. Viswanathan, L. Timmermann, S.B. Eickhoff, G.R. Fink, C. Grefkes, Dopaminergic modulation of motor network dynamics in Parkinson's disease, Brain : a journal of neurology 138(Pt 3) (2015) 664-78.

[61] M. Hallett, The intrinsic and extrinsic aspects of freezing of gait, Movement disorders : official journal of the Movement Disorder Society 23 Suppl 2 (2008) S439-43.

[62] W. Bouthour, P. Megevand, J. Donoghue, C. Luscher, N. Birbaumer, P. Krack, Biomarkers for closedloop deep brain stimulation in Parkinson disease and beyond, Nature reviews. Neurology 15(6) (2019) 343352.

[63] N.C. Swann, C. de Hemptinne, M.C. Thompson, S. Miocinovic, A.M. Miller, R. Gilron, J.L. Ostrem, H.J. Chizeck, P.A. Starr, Adaptive deep brain stimulation for Parkinson's disease using motor cortex sensing, Journal of neural engineering 15(4) (2018) 046006. 